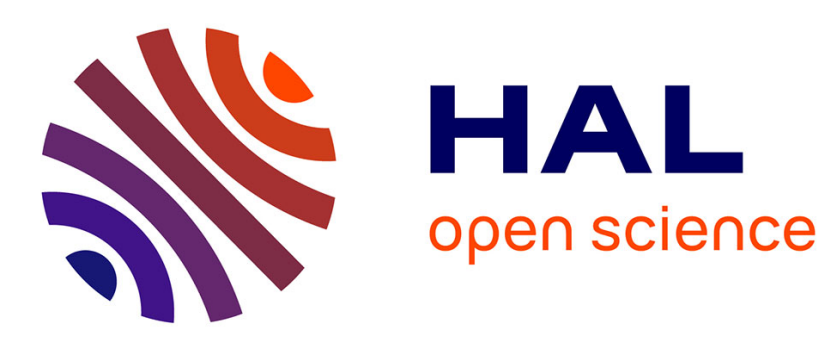

\title{
Market sentiments and convergence dynamics in decentralized assignment economies
}

\author{
Bary S R Pradelski, Heinrich H Nax
}

\section{To cite this version:}

Bary S R Pradelski, Heinrich H Nax. Market sentiments and convergence dynamics in decentralized assignment economies. International Journal of Game Theory, 2020, 49 (1), pp.275-298. 10.1007/s00182-019-00694-0 . hal-03100116

\section{HAL Id: hal-03100116 https://hal.science/hal-03100116}

Submitted on 6 Jan 2021

HAL is a multi-disciplinary open access archive for the deposit and dissemination of scientific research documents, whether they are published or not. The documents may come from teaching and research institutions in France or abroad, or from public or private research centers.
L'archive ouverte pluridisciplinaire HAL, est destinée au dépôt et à la diffusion de documents scientifiques de niveau recherche, publiés ou non, émanant des établissements d'enseignement et de recherche français ou étrangers, des laboratoires publics ou privés. 


\title{
Market sentiments and convergence dynamics in decentralized assignment economies
}

\author{
Bary S.R. Pradelski Heinrich H. Nax
}

Received: date / Accepted: date

\begin{abstract}
In two-sided markets with transferable utility ('assignment games'), we study the dynamics of trade arrangements and price adjustments as agents from the two market sides stochastically match, break up, and re-match in their pursuit of better opportunities. The underlying model of individual adjustments is based on the behavioral theories of adaptive learning and aspiration adjustment. Dynamics induced by this model converge to approximately optimal and stable market outcomes, but this convergence may be (exponentially) slow. We introduce the notion of a 'market sentiment' that governs which of the two market sides is temporarily more or less amenable to price adjustments, and show that such a feature may significantly speed up convergence.
\end{abstract}

Keywords assignment games $\cdot$ core $\cdot$ evolutionary game theory $\cdot$ matching markets $\cdot$ convergence time $\cdot$ market psychology 


\section{Motivation}

This paper is concerned with trade dynamics in decentralized two-sided economies described by the assignment game (Shapley and Shubik, 1972). Assignment games describe two-sided markets, where individual valuations for buying and selling may depend on the identities of each trade counterparty, possibly with full heterogeneity. As discussed in Shapley and Shubik (1972), several widespread types of markets are special cases of such games. In the 'house market', for example, buyers may have heterogenous buy valuations, while sellers may have sell valuations that are independent of the buyers' identities. The single-item economy is also a special case, where both sides' valuations are independent of the counterparty's identity. ${ }^{1}$ Consequently, the assignment game belongs to the classical canon of market games. Relevant theory is extensively surveyed in Núñez and Rafels (2015).

Building on own prior work, which we presented in Nax and Pradelski (2015), this paper is concerned with decentralized dynamics played on the assignment game. The behavioral dynamics that underly these dynamics are induced by a model of individual adjustments where market participants continue to seek new trade opportunities and re-negotiate in order to improve their own shares of the gains of trade. The learning theories underlying this model have a long tradition in economics and psychology, and were motivated in detail in Section 2 of our aforementioned prior paper.

In essence, the key drivers of individual behavior belonging to this class of models are own prior market experiences that trigger directional bid/ask adjustments. In particular, market participants occasionally adjust their bids/asks in a way that would generate higher payoffs for them in the present trading period:

[ + ] traders whose current bids/asks lead to trade occasionally make slightly more aggressive bids/asks in the hope of making better deals with another trade partner

[ - ] traders whose current bids/asks do not lead to trade submit less aggressive bids/asks in the hope of attracting a trade partner

The individual-level adjustments thus formulated result in stochastic sequences of adjustments to trade arrangements and to trading prices. Akin to Roth and Vande Vate (1990)'s result that random paths of 'blocking pairs' implement stability in the marriage market (Gale and Shapley, 1962), we have shown in Nax and Pradelski (2015) that the family of dynamics outlined above (in terms of $[+]$ and $[-]$ ) converges to optimality in terms of trade arrangements as well as to stability in terms of trading prices. Related results were obtained by Chen et al. (2016), Klaus and Payot (2015), Biró et al. (2012), and Bayati et al. (2015), with some work also considering the speed of convergence in non-transferable utility matching markets (Ackermann et al., 2011).

In the present paper, we investigate the consequences - in terms of convergence - of introducing 'market sentiments' that influence individual behavior beyond the purely personal experience. Market sentiments are assumed to put pressure on one or the other side of the market. Consequently, while agents continue to rely on own trade experiences (as in our original model), they now also take market sentiments into account such that agents from their own market side are currently either 'flexible' (willing to reduce bids and aspirations) or 'sticky' (unwilling to reduce). These market sentiments are inspired by economic models of business cycles and resulting labor market flexibilities, as used in some applied work.

The main contributions of our paper are to show that the resulting dynamics lead to approximately stable and optimal market outcomes, and that market sentiments may speed up con-

\footnotetext{
${ }^{1}$ As an example of such a market, Shapley and Shubik discuss the famous 'Böhm-Bawerk (horse) market' (von Böhm-Bawerk, 1891).
} 
vergence which otherwise may be exponentially slow. These findings present a first step towards understanding the role of market sentiments in influencing convergence phenomena, and indicate some new avenues for future empirical and theoretical research.

The remainder of this paper is structured as follows. Next, we introduce the model and our dynamics. Section 3 studies the dynamic with market sentiment, and Section 4 the dynamic without market sentiment. Section 5 concludes.

\section{Model}

In this section, we shall develop the cooperative formulation of the assignment game, detail the dynamic components of our behavioral model, and illustrate how our dynamics play out in an example.

\subsection{The assignment game}

Firms $i \in \mathcal{F}$ and workers $j \in \mathcal{W}$ are two populations of agents representing the two market sides. (Note that instead of firms and workers we could be speaking of buyers and sellers.) Let $V=\mathcal{F} \cup \mathcal{W}$. We assume that $|\mathcal{F}|=|\mathcal{W}|=N$. (Note that this assumption is without loss of generality since we can always add 'dummy' agents to the smaller side of the market.) The game is played repeatedly. At any given point in time, a player can be matched to at most one player. A player currently not matched to another player is called single.

\subsubsection{Gains of trade}

Reservation value. Each firm $i$ has a reservation value, $r_{i}^{+}(j)$ for being matched with worker $j$. That is, firm $i$ is willing to pay at most $r_{i}^{+}(j)$ when matching with worker $j$ and otherwise prefers to remain single. Similarly each worker $j$ is willing to accept no less than reservation value $r_{j}^{-}(i)$ when matching with firm $i$ and otherwise prefers to remain single.

We consider the reservation values to be private information that are unknown to other agents. Other than in a standard single-item economy, these heterogeneous match values express heterogeneous preferences for trading with different counterparties. For example, potential realestate buyers may have different valuations for different properties on the market. Depending on application one can simplify the assignment game by associating to one or both sides simpler valuation structures.

Match value. We shall assume that players are individually rational and therefore only allow matches that yield strictly positive gains of trade. If for $i, j, r_{i}^{+}(j)>r_{j}^{-}(i)$ holds, then the gains of trade between the two, which we denote as the match value between $i$ and $j$, are defined by $w_{i j}=r_{i}^{+}(j)-r_{j}^{-}(i)$. Otherwise, $w_{i j}=0$. Let $W=\left(w_{i j}\right)_{i \in \mathcal{F}, j \in \mathcal{W} \text { and } r_{i}^{+}(j)>r_{j}^{-}(i)}$ be the matrix of match values.

Let $w^{*}=\max _{i, j}\left\{w_{i j} \mid r_{i}^{+}(j)>r_{j}^{-}(i)\right\}$ be the maximal match value amongst all pairs. It will be convenient to define the associated game graph $G=(V, E)$ where $V=\mathcal{F} \cup \mathcal{W}$, and vertices $i$ and $j$ are connected by an edge $e_{i j} \in E$ with weight $w_{i j}$ if $r_{i}^{+}(j)>r_{j}^{-}(i)$. Note that because reservation values are private information the match values are hidden variables unknown to the players. 


\subsubsection{Outcomes}

Since we will be interested in dynamic processes we introduce time steps $t=0,1,2,3, \ldots$. At each time step $t$, an outcome is described by three ingredients:

Matching. The matching at time $t$ is described by $M^{t}=\left(m_{i j}^{t}\right)_{i \in \mathcal{F}, j \in \mathcal{W}} \in\{0,1\}^{\mathcal{F} \times \mathcal{W}}$ such that each row and each column of $M^{t}$ sums to at most 1.

Prices. When $i$ is matched with $j$ at time $t$ they trade at a unique price, $\pi_{i j}^{t}$, which must lie $\in\left[r_{j}^{-}(i), r_{i}^{+}(j)\right]$ given their reservation values if $w_{i j}>0 . \pi^{t}$ describes the vector of realized prices.

Payoffs. If $i$ and $j$ are matched with each other, we refer to the individual share of the common gains of trade by the payoff, which to firm $i$ is $\phi_{i}^{t}=r_{i}^{+}(j)-\pi_{i j}^{t}$ and to worker $j$ is $\phi_{j}^{t}=\pi_{i j}^{t}-r_{j}^{-}(i)$. If player $i$ is single his payoff -assuming he/she consumes her reservation value - is $\phi_{i}^{t}=0$. Let $\Phi^{t}=\left(\phi_{i}^{t}\right)_{i \in V}$.

Note that even if prices are public information, that payoffs remain private information because reservation values are private information.

\subsubsection{Cooperative representation}

Given the above ingredients, the assignment game as a cooperative game $G(\nu, V)$ is defined as follows. Let $\nu: \mathcal{P}(V) \rightarrow \mathbb{R}$ such that

$-\nu(i)=\nu(\emptyset)=0$ for all singletons $i \in V$

$-\nu(\{i, j\})=w_{i j}$ for all $\{i, j\} \in \mathcal{F} \times \mathcal{W}$

$-\nu(S)=\max _{P \in \operatorname{perm}\left(1, \ldots, k^{\mathcal{F}}\right), Q \in \operatorname{perm}\left(1, \ldots, k^{\mathcal{W}}\right)}\left\{\nu\left(\left\{i_{P(1)}, j_{Q(1)}\right\}\right)+\ldots+\nu\left(\left\{i_{P(k)}, j_{Q(k)}\right\}\right)\right\}$ for all $S=\left(i_{1}, i_{2}, \ldots, i_{k^{\mathcal{F}}}, j_{1}, j_{2}, \ldots, j_{k^{\mathcal{W}}}\right) \subseteq V$ and $k=\min \left\{k^{\mathcal{F}}, k^{\mathcal{W}}\right\}$

where $\operatorname{perm}(1, \ldots, k)$ is the set of all permutations of the numbers $1, \ldots, k$. The number $\nu(V)$ specifies the value of an optimal assignment (as defined below).

\subsubsection{The core}

We shall now define optimality and stability properties of outcomes of the assignment game:

Optimality. A matching $M$ is optimal if $\sum_{i, j \in V} m_{i j} \cdot w_{i j}$ is maximal.

Pairwise stability. A payoff vector $\Phi$ is pairwise stable if for all $i, j \in V: \phi_{i}+\phi_{j} \geq w_{i j}$.

Shapley and Shubik (1972) showed that the core of the assignment game is always nonempty and is characterized by pairwise stability:

Core. A matching $M$ together with the payoffs $\Phi$ is in the core if and only if $M$ is optimal and $\Phi$ is pairwise stable.

We shall focus on the approximate core:

$\varepsilon$-Core. A matching $M$ together with the payoffs $\Phi$ is in the $\varepsilon$-core if and only if $M$ is optimal and $\Phi$ is $\varepsilon$-pairwise stable, that is, for all $i, j \in V: \phi_{i}+\phi_{j} \geq w_{i j}-\varepsilon$.

\subsubsection{Dynamic variables}

So far we introduced the assignment game as a static game without any additional components. We shall now introduce the dynamic components of our model (before describing -in the next section- how they evolve). 
Aspiration levels. At the end of any step $t=0,1,2, \ldots$, each player $i \in V$ has an aspiration level, $d_{i}^{t} \geq 0$, which determines the minimal payoff at which he is willing to be matched. Let $\mathbf{d}^{t}=\left(d_{i}^{t}\right)_{i \in V}$.

Feasibility. A pair of aspiration levels $\left(d_{i}^{t}, d_{j}^{t}\right)$ is feasible, expressing whether both players would be willing to enter a partnership involving the two players, if

$-r_{i}^{+}(j)-d_{i}^{t}>r_{j}^{-}(i)+d_{j}^{t}$ (equivalently $\left.d_{i}^{t}+d_{j}^{t}<w_{i j}\right)$,

$-r_{i}^{+}(j)-d_{i}^{t}=r_{j}^{-}(i)+d_{j}^{t}$ (equivalently $d_{i}^{t}+d_{j}^{t}=w_{i j}>0$ ) and one of the players is single, or

$-i$ and $j$ are currently in a partnership (i.e., are matched to each other).

We shall say that the pair between $i$ and $j$ is feasible in period $t$ if $\left(d_{i}^{t}, d_{j}^{t}\right)$ is feasible.

Market sentiment and price flexibility/stickiness Market sentiment is an exogenous binary random variable, $\Psi^{t} \in\{\ominus, \oplus\}$, that acts as a correlation device determini ng which players currently exhibit price flexibility and which players currently exhibit price stickiness. If market sentiment is firm-favoring $\left(\Psi^{t}=\ominus\right)$, for example in periods of high unemployment, workers are price flexible and firms are price sticky. If market sentiment is worker-favoring $\left(\Psi^{t}=\oplus\right)$, for example in periods of low unemployment, firms are price flexible and workers are price sticky. We shall later assume that $\Psi^{t}$ switches at a fixed rate of $\Theta\left(N^{2+c}\right)$ time steps, for some fixed $c \geq 0 .^{2}$

No feasible alternative. Suppose $i$ and $j$ are feasible. Then say that player $j$ has no feasible alternative to $i$ if for all $i^{\prime} \neq i, i^{\prime}$ and $j$ are not feasible. Otherwise say that $j$ has a feasible alternative to $i$.

An agent who is price sticky only agrees to a match if the price is such that his payoff will be at least as high as his aspiration level. On the other hand, an agent $j$ who is price flexible will be less tough in negotiations with agent $i$ if $j$ has no feasible alternative to $i$. In this case $j$ may agree to a price such that his payoff is a bit below his aspiration level. This will be formalized in the next section.

State. The state at the end of period $t$ is given by the matching, the aspiration levels, and market sentiment: $\left[M^{t}, \mathbf{d}^{t}, \Psi^{t}\right]$.

\subsubsection{Class of games}

We restrict much of our analysis to games that have the following property, called 'tie-freeness':

Definition 1. Say that an assignment game is 'tie-free' if all subgraphs of the corresponding bipartite graph have a unique optimal matching. That is, there is no sequence of firms and workers $\left(a_{1}, b_{1}, a_{2}, b_{2}, \ldots, a_{k}, b_{k}\right)$, where $\left\{a_{1}, \ldots, a_{k}\right\} \subseteq \mathcal{F},\left\{b_{1}, \ldots, b_{k}\right\} \subseteq \mathcal{W}$ such that:

$$
w_{a_{1} b_{1}}>0, w_{a_{2} b_{2}}>0, \ldots, w_{a_{k} b_{k}}>0 \text { and } w_{a_{1} b_{k}}>0, w_{a_{2} b_{1}}>0, \ldots, w_{a_{k} b_{k-1}}>0
$$

and

$$
w_{a_{1} b_{1}}+w_{a_{2} b_{2}}+\ldots+w_{a_{k} b_{k}}=w_{a_{1} b_{k}}+w_{a_{2} b_{1}}+\ldots+w_{a_{k} b_{k-1}} .
$$

\footnotetext{
${ }^{2}$ Note that this implies that the market sentiment changes as slow as $\Theta\left(N^{2}\right)$ or slower but not exponentially slow.
} 
Tie-freeness holds almost surely for assignment games where, for example, match values are drawn from atomless independent distributions. Further, note that tie-freeness holds in particular for any assignment game where the set of positive-weight edges constitutes a tree since then Equation (1) never holds.

As shown by Klaus and Payot (2015) convergence to the core in finite time can only be guaranteed when the game is discretized. We shall thus consider discretized games that are tie-free. For a given tie-free game, round all match values, aspiration levels, and prices to the closest multiple of some $\delta$, where we choose $\delta>0$ to be maximal such that the resulting discretized game still is tie-free. ${ }^{3}$

Consequently, our results regarding the rate of convergence to core outcomes will depend on the discretization unit $\delta$. Intuitively, game graphs where many matches are individually rational will require a finer discretization than game graphs where few matches are individually rational. Sections 3 discusses the scaling of $\delta$ and its effect on the rate of convergence in detail.

\subsection{Dynamic play}

At the beginning of every stage $t$, one player - uniformly chosen at random- is activated: The active agent scans the other side of the market, looking for a feasible match. For example, consider a job-list available online. If there is a feasible match he picks one at random and matches with him. The price is set at random satisfying the conditions determined by the aspiration levels and price stickiness. We can think of the price as the outcome of a bargaining process that we do not model explicitly. At the end of each period the two players adapt their aspiration levels to equal their new payoffs. If there is no feasible match and the active player is matched the state remains the same. If there is no feasible match and the active player is single he remains single and reduces his aspiration level.

Formally, let $\left[M^{0}, \mathbf{d}^{0}, \Psi^{0}\right]$ be any initial state. Suppose that in period $t+1(t \geq 0)$ firm $i$ is activated (the dynamic is symmetric if a worker is activated).

If there exists a feasible match for $i, i$ picks one such player at random, matches with him and the price $\pi_{i j}^{t+1}$ is set at random between

$$
r_{j}^{-}(i)+d_{j}^{t}-\Delta_{j}(i) \text { and } r_{i}^{+}(j)-d_{i}^{t},
$$

where

$$
\Delta_{j}(i)= \begin{cases}\delta & \text { if } j \text { is price flexible and has no feasible alternative } \\ 0 & \text { else }\end{cases}
$$

and if $\Delta_{j}(i)=\delta$ the price is set at $r_{j}^{-}(i)+d_{j}^{t}-\Delta_{j}(i)$ with strictly positive probability, bounded away from zero by a constant. At the end of the period the aspiration levels of the newly matched pair $(i, j)$ are set to their new realized payoffs,

$$
\left.\left.d_{i}^{t+1}=\phi_{i}^{t+1}=\left(r_{i}^{+}(j)-\pi_{i j}^{t+1}\right)_{+} \quad \text { and } \quad d_{j}^{t+1}=\phi_{j}^{t+1}=\right) \pi_{i j}^{t+1}-r_{j}^{-}(i)\right)_{+},
$$

and the matching $M^{t+1}$ is updated accordingly, that is, $m_{i j}^{t+1}=1, m_{k l}^{t+1}=0$ for all $k \neq i$ with $l=j$ and for all $l \neq j$ with $k=i$.

\footnotetext{
${ }^{3}$ Such $\delta$ always exists since the rational numbers are dense in the real numbers. Hence, one can choose $\delta$ small enough to approximate the match values to any desired degree and thus sustain tie-freeness.
} 
If there is no feasible match for player $i$, if $i$ was previously matched he stays with his previous partner and keeps his payoff and thus aspiration level. Otherwise $i$ remains single and reduces his aspiration level:

$$
d_{i}^{t+1}=d_{i}^{t}-\delta
$$

See Figure 1 for illustrations.

Fig. 1: Transition diagram for active, matched (left) and active, single agent (right).

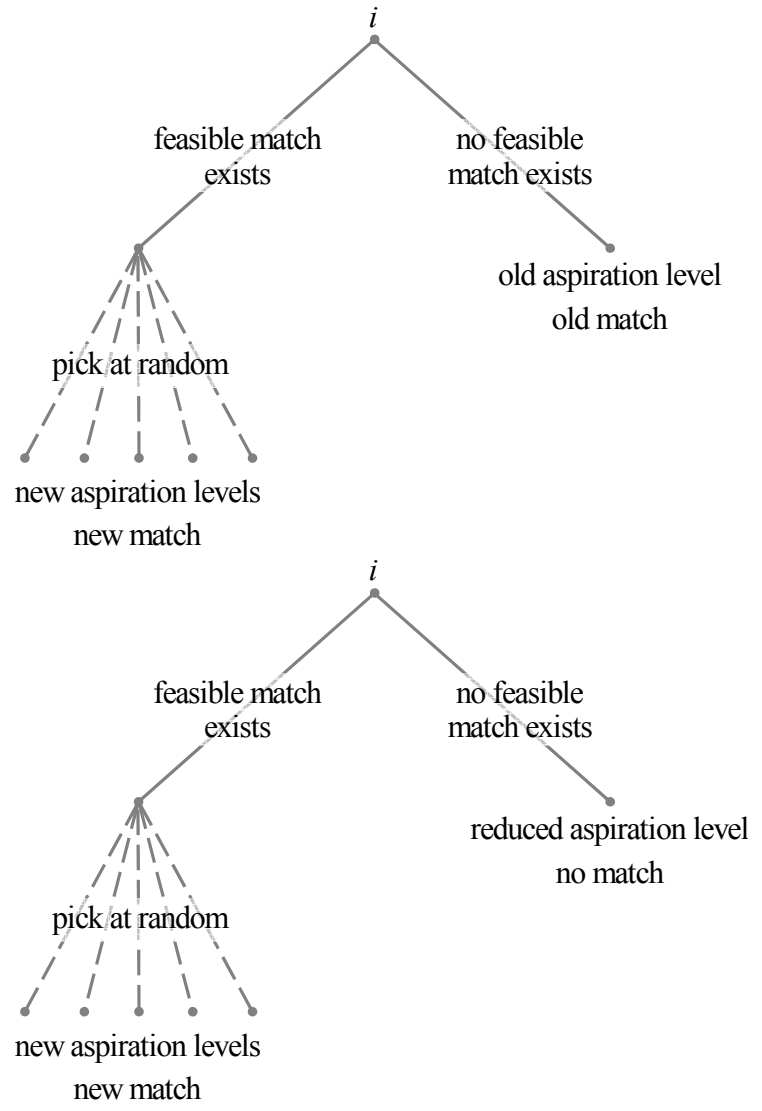

Note that, market and information decentralization hold. For market decentralization, agents interact at random points in time and prices are set at random with weak boundary conditions which are natural from the point of view of each player. For information decentralization, in each step players only know their own reservation values, aspiration level, and the price they trade at. They do not know other players' reservation values or aspiration levels.

Finally, given the updating rule, the payoffs and prices are deducible from the aspiration levels and the matching. For ease of exposition we shall focus on the latter.

\subsection{Example}

Let $V=\mathcal{F} \cup \mathcal{W}=\left\{i_{1}, i_{2}\right\} \cup\left\{j_{1}, j_{2}\right\}$ and $w_{11}=5, w_{12}=w_{21}=7, w_{22}=10$. Let $\delta=1$. 


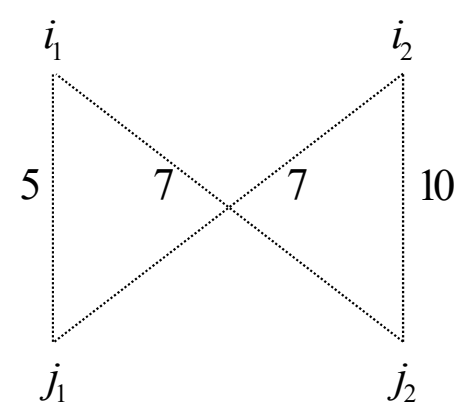

In all figures a dotted edge between two players $i$ and $j$ indicates that $w_{i j}>0$, a black solid edge that they are matched $\left(m_{i j}^{t}=1\right)$, and a black dashed edge that $d_{i}^{t}+d_{j}^{t}=w_{i j}^{t}>0$ and $i$ and $j$ are not matched in period $t$. We shall call such a dashed edge tight. Numbers next to edges indicate match values. The current aspiration levels are shown next to the name of an agent.

One can verify that the optimal matching is unique and such that firm $i_{1}$ matches worker $j_{1}$ and $i_{2}$ matches $j_{2}$.

\section{period t: Current state}

Suppose that, at the end of some period $t,\left(i_{2}, j_{1}\right)$ are matched, $i_{1}$ and $j_{2}$ are single, and $i_{2}$ and $j_{2}$ share a tight edge.

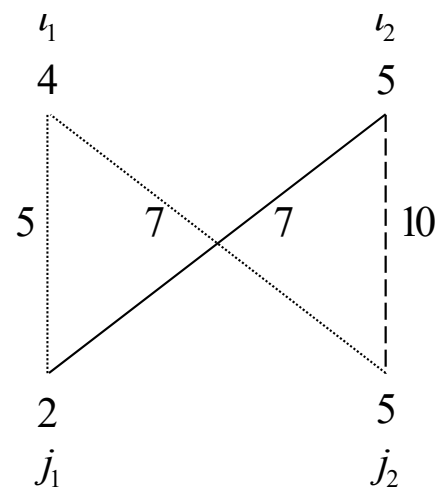

Note that the aspiration levels satisfy $d_{i}^{t}+d_{j}^{t} \geq w_{i j}$ for all $i$ and $j$, but the assignment is not optimal and the payoffs are not stable.

period $t+1: \quad$ Activation of single agent $i_{1}$ and firm-favoring market sentiment $\Psi^{t}=\ominus$

Suppose that $i_{1}$ is activated in period $t+1$ and market sentiment is currently firm-favoring. $i_{1}$ 's current aspiration level is too high in order to be feasible with any worker. Hence he remains single and reduces his aspiration level by 1 . 


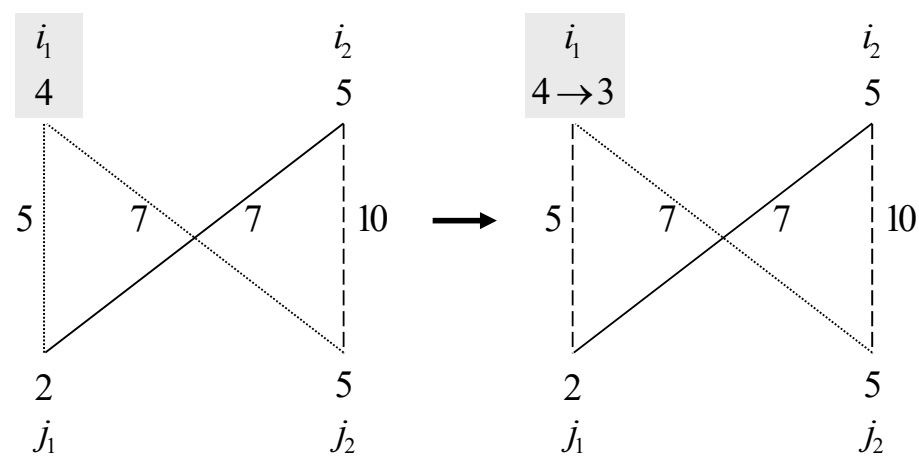

period $t+2: \quad$ Activation of matched agent $j_{1}$ and worker-favoring market sentiment $\Psi^{t+1}=\oplus$ Suppose that $j_{1}$ is activated in period $t+2$ and market sentiment has changed and it is now worker-favoring. $i_{1}$ and $j_{1}$ is the only feasible pair involving $j_{1}$. They thus match and given that $i_{1}$ has no feasible alternative and is price flexible (since market sentiment is currently worker-favoring) the price, with positive probability, is set such that $j_{1}$ increases his aspiration level by $\delta=1$.

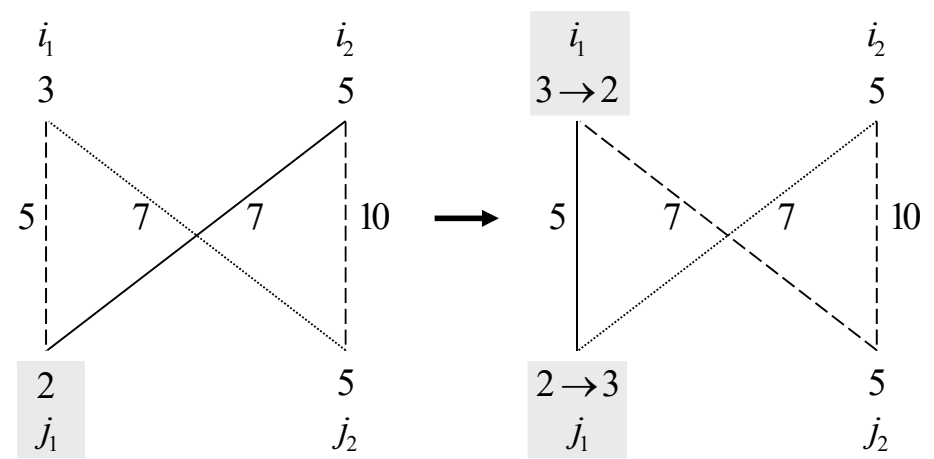

period $t+3: \quad$ Activation of single agent $i_{2}$ and firm-favoring market sentiment $\Psi^{t+2}=\ominus$ Suppose that $i_{2}$ is activated in period $t+3$ and market sentiment has changed again is now firm-favoring. $i_{2}$ and $j_{2}$ is the only feasible pair involving $i_{2}$. They thus match and although workers are currently price flexible (market sentiment is firm-favoring), $j_{2}$ has a feasible alternative (namely $i_{1}$ ) and thus is not accepting less than his aspiration level. They are matching at their current aspiration levels. 


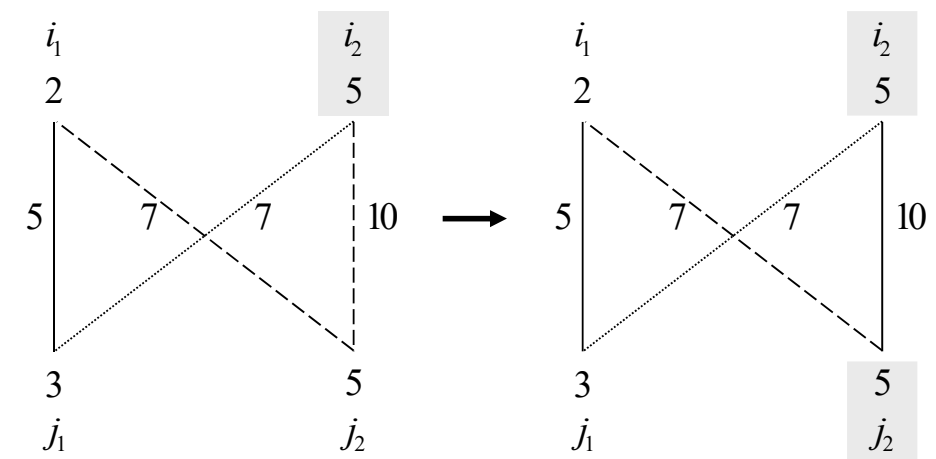

Note that the resulting state is in the core.

\section{Results with market sentiment}

We shall now proceed to establish convergence and bounds for the rate of convergence under the assumption of tie-freeness and in the presence of a fluctuating market sentiment.

In terms of notation, recall that $\nu(V)$ is the value of an optimal assignment, $w^{*}$ is the maximal match value, and $\delta$ is the unit of discretization. Concerning the asymptotic notations, we shall write $f(N)=O(g(N))$ if $f(N)<C \cdot g(N)$ for some $C>0$ constant and $N$ sufficiently large, and $f(N)=\Theta(g(N))$ if there exist two constants $k, K \geq 0$ and a positive integer $N_{0}$ such that $k g(N) \leq f(N) \leq K g(N)$ for all $N \geq N_{0}$.

Proposition 2. Given a discretized tie-free assignment game, suppose that $\Psi^{t}$ switches at a fixed polynomial rate of $\Theta\left(N^{2+c}\right)$ ( $c \geq 0$ fixed) time steps. From any starting state such that for all $i, d_{i} \leq w^{*}$, the expected convergence time to the core (of the discretized game) is given by

$$
O\left(N^{2+c} \cdot \max \left\{N^{2}, \frac{1}{\delta}\left(N \cdot w^{*}-\nu(V)\right)\right\}\right)
$$

iterations. The total computational complexity is

$$
O\left(N^{4+c} \cdot \max \left\{N^{2}, \frac{1}{\delta}\left(N \cdot w^{*}-\nu(V)\right)\right\}\right) .
$$

The proposition states that, for any discretized tie-free assignment game, the convergence time can be bounded above by the number of players and the discretization unit $\delta$ if the market sentiment switches at certain rates. In particular, it needs to switch with times that are polynomial in $N$, but at least quadratically. The economic interpretation of the result would be that, in order for a market to perform well, the market must be subject to a certain degree of sentiment fluctuations but not too much. This notion is related to the idea of public signals correlating play and thus speeding up rates of convergence as introduced in Lewis (1969), which has also been used in Newton and Angus (2015) and Arieli and Young (2016). If fluctuations are either faster or exponentially slow in $N$ convergence rates will no longer be bounded as in the proposition.

Next, we proceed to the proof of the proposition, which we carry out via several intermediate Lemmas. First 
, note that the process is fully characterized by the state variables $M^{t}, d^{t}$, and $\Psi^{t}$, where $d^{t}$ together with the matching determines realized payoffs and prices. We shall thus only focus on the changes in the state variables. We introduce notation in the spirit of Nax and Pradelski (2015). An edge between player $i$ and $j$ is called tight if $d_{i}^{t}+d_{j}^{t}=w_{i j}$ and loose if $d_{i}^{t}+d_{j}^{t}<$ $w_{i j}$. Given aspiration levels $\mathbf{d}^{t}$, if for all $i, j, d_{i}^{t}+d_{j}^{t} \geq w_{i j}$ and for each $i$, either there exists $j$ such that $d_{i}^{t}+d_{j}^{t}=w_{i j}$ or $d_{i}^{t}=0$ we shall call $\mathbf{d}^{t}$ good. Let a tight component be a maximal subgraph of players connected by tight edges.

In the following lemma we show that once a state is reached such that no loose edges exist all future states will not have loose edges. We give a bound for the waiting time of $O\left(N^{2} \log N\right)$. This crude bound is obtained by noting that two players, if they meet once, will never again share a loose edge. Thus it suffices that all pairs meet at least once.

Lemma 3. Starting from any initial state $\left[M^{0}, \mathbf{d}^{0}, \Psi^{0}\right]$, the expected time until the dynamic reaches a state such that for all $i, j d_{i}+d_{j} \geq w_{i j}$ is $O\left(N^{2} \log N\right)$. Once for all $i, j d_{i}+d_{j} \geq w_{i j}$ holds for some $T \geq 0$ it holds for all $t \geq T$.

Proof We define

$$
\begin{aligned}
U^{t} & =\left\{(i, j): i \in \mathcal{F}, j \in \mathcal{W} \text { and } d_{i}^{t}+d_{j}^{t}<w_{i j}\right\} \\
S^{t} & =\{(i, j): i \in \mathcal{F}, j \in \mathcal{W} \text { and } i \text { and } j \text { are feasible at } t\}
\end{aligned}
$$

It follows from the updating rule that if, for any $i \in \mathcal{F}, j \in \mathcal{W}$ and for some $T, d_{i}^{T}+d_{j}^{T} \geq$ $w_{i j}$ holds, then for all $t \geq T, d_{i}^{t}+d_{j}^{t} \geq w_{i j}$, proving the second part of the Lemma. Further this shows that the set $U^{t}$ is non-increasing and thus we are interested in the expected time until $U^{t}=\emptyset$ for the first time. It is easy to see that $U^{t} \subseteq S^{t}$ and, by the updating rule, the probability for a feasible pair to match in a given period is lower bounded by $1 / N^{2}$. We thus have $\forall(i, j) \in U^{t}$ :

$$
\mathbb{P}\left((i, j) \notin U^{t+1}\right) \geq \frac{1}{N^{2}}
$$

Further note that $\forall(i, j) \notin U^{t}$ :

$$
\mathbb{P}\left((i, j) \notin U^{t+1}\right)=1 \geq \frac{1}{N^{2}}
$$

Hence a crude bound is to assume equality and ask how long it takes until each pair is picked at least once. Now we can use an analogy to the Coupon Collector's Problem (see, for example, Feller (1950)). The Coupon Collector's Problem asks the following question: Suppose you have $x$ coupons, what is the expected time until you have picked each coupon at least once when picking with replacement. The answer is that the number of trials needed is $O(x \log x)$. Thus the expected time until each pair of players $\left(N^{2}\right)$ has been selected at least once is $O\left(N^{2} \log N\right)$ (given that among feasible matches a match is selected uniformly at random).

Next, we show that given we have reached the set of states with no loose edges the sum of aspiration levels is non-increasing in all future states.

Lemma 4. Let $D^{t}:=\sum_{i \in V} d_{i}^{t}$. Suppose the process is in a state as in Lemma 3 at time $T \geq$ 0 . Then $D^{t}$ is non-increasing for all $t \geq T$.

Proof Given $D^{t}, D^{t+1}$ can only increase if two players, say $i, j$, newly match and $d_{i}^{t}+d_{j}^{t}<w_{i j}$. But given we are in a state as in Lemma 3 , for all $i, j d_{i}^{t}+d_{j}^{t} \geq w_{i j}$ and hence we conclude that $D^{t+1} \leq D^{t}$ 
We go on to bound the time until the sum of aspiration levels is minimal provided that there are no loose edges (Lemmas 5 and 6 ). We show that the minimum will be equal to the value of the game, $\nu(V)$. The proof of this result makes use of alternating market sentiment and the assumption that the game is tie-free (see Definition 1). By tie-freeness all tight components are trees. If all tight components allow perfect matchings we are done. ${ }^{4}$ Otherwise we show how in a given tight component which does not allow a perfect matching the sum of aspiration levels can be reduced. We use the market sentiment to prove that the expected time until the sum of aspiration levels is equal to the value of the game is $O\left(N^{2+c} \cdot \frac{1}{\delta} \cdot\left(N \cdot w^{*}-\nu(V)\right)\right.$. Note that once we are in such a state we will always remain in the set of states with no loose edges and the sum of aspiration levels equal to the value of the game (by Lemmas 3 and 4 ).

We shall first recall a folk result regarding the existence of perfect matchings on trees (see, for example, Edmonds (1965)).

Support Lemma A. A tree $C$ admits a perfect matching (a matching such that each player is matched) if and only if for every vertex $v$, the graph $C-v$ has exactly one odd sub-component.

Proof First, suppose there exists a perfect matching in $C$. Let $u$ be the vertex matched with $v$ in the perfect matching. Then each component in $C-v$ not containing $u$ must have a perfect matching and is thus even. Finally the component containing $u$ must be odd.

Second, suppose that for every vertex $v$ the graph $C-v$ has exactly one odd component. We shall construct a perfect matching. Given $C$ is a tree and by our assumption each vertex $v$ has a unique neighbor in one component of odd size, say $u$. We shall show that pairing each $v$ to its neighbor $u$ in the odd component yields a perfect matching. It suffices to show that if $u$ is the neighbor of $v$ in the unique odd component of $C-v$ (say odd $(C-v)$ ) that it is also the case that $v$ is the neighbor of $u$ in the unique odd component of $C-u$ (say odd $(C-u)$ ). Note that the component of $C-u$ which contains $v$ is disjoint with odd $(C-v)$. But then it follows that $v$ is part of a component which is comprised of even components in $C-v$. Adding $v$ it follows that $v$ must be in an odd component $\operatorname{odd}(C-u)$ which by assumption is unique.

Note that if the unique optimal matching is not perfect we can transform the game such that the unique optimal matching will be perfect and the core has not changed. To do so add for any player who is single in the unique optimal matching a player on the other side of the market with match value $\delta$ towards that player and no other possible matches. Clearly the core of the original game does not change through these operations. Suppose from now on that the optimal matching is perfect.

Lemma 5. Given a state as in Lemma 3, either the expected waiting time until $D^{t}$ decreases by at least $\delta$ is $O\left(N^{2+c}\right)$ or $D^{t}=\nu(V)$.

Proof First, suppose there exists a player $i$ such that he has no tight edge, that is for all $j$ $d_{i}^{t}+d_{j}^{t}>w_{i j}>0$. The expected time until $i$ is selected is $O(N)$. If the condition still holds he reduces his aspiration level and no other player increases his aspiration level, hence $D$ has decreased.

Now suppose that for all players $i$, there exists $j$ such that $d_{i}^{t}+d_{j}^{t}=w_{i j}>0$. Let a tight component, $C^{t}$, be a maximal subgraph of players (nodes) connected by tight $\left(d_{i}^{t}+d_{j}^{t}=w_{i j}>0\right)$ edges. Let $C_{1}^{t}, C_{2}^{t}, \ldots, C_{k}^{t}$ be all of the tight components. By assuming tie-freeness the tight

\footnotetext{
${ }^{4}$ We say that a matching is perfect when all players in a given component are matched.
} 
components are trees. This is the case because otherwise there exists a cycle in a given component such that two alternating partitions have the same sum of match values, thus contradicting tie-freeness (by satisfying both conditions in Definition 1: Equation (1), since tight edges are assumed to have positive match values, and Equation (2) by above argument).

We shall show that either there exists a perfect matching (a matching such that each player is matched) within each component or there exists at least one component such that the sum of aspiration levels within the component can be reduced by at least $\delta$ independently of the other components.

First, suppose that all components have a perfect matching. It follows that $D^{t}=\nu(V)$ and we are done.

Now, suppose that there exists a component $C^{t}$ which has no perfect matching. In the following we describe a series of 'operations' that yield a reduction in the aspiration levels. We shall describe the dynamics and the expected waiting time later.

Suppose, $\#\left\{i \in C^{t}: i \in \mathcal{F}\right\} \neq \#\left\{i \in C^{t}: i \in \mathcal{W}\right\}$, that is, there are either more workers or more firms. A reduction of aspiration levels by 1 by the players on the larger side and an increase by 1 by the players on the smaller side yields a state where $D$ has decreased. Note that after the increases and reductions $\mathbf{d}$ is still good, given the reductions are only 1 .

Next, suppose $\#\left\{i \in C^{t}: i \in \mathcal{F}\right\}=\#\left\{i \in C^{t}: i \in \mathcal{W}\right\}$. By Support Lemma A a tree $C$ has a perfect matching if and only if for every vertex $v$, the graph $C-v$ has exactly one odd sub-component. Hence, there exists a vertex $v$ such that $C^{t}-v$ has at least 3 odd sub-components. (Given that the number of firms is equal to the number of workers there must be an odd number of odd sub-components. Thus we can dispense with the case of zero or two odd sub-components.) Without loss of generality, let $v$ be a firm. Denote the odd subcomponents resulting from $C-v$ by $C_{o d d 1}^{t}, C_{o d d 2}^{t}, \ldots$. In each of these sub-components there are either strictly more firms or strictly more workers. In particular given there are at least 3 odd sub-components at least one of them contains more firms than workers. For the latter subcomponent aspiration level reductions by $\delta$ by all firms and aspiration level increases by $\delta$ by all workers yield a decrease of $D$, while $\mathbf{d}$ remains good.

It is important to note that these adjustments can only take place if the players on the reducing side initially have positive aspiration levels. This might not be the case for all such constructions, but it will always be the case for at least one. This follows by the proof in Nax and Pradelski (2015). They show that the core can be reached by only following transitions as described above or by rematchings within tight component with non-changing aspiration levels. But note that in our model rematchings are not necessary to make transitions as described above possible. Hence, by the proof of Theorem 1 in Nax and Pradelski (2015), if there is no tight component allowing an aspiration level reduction (that is the larger side of a component having all positive aspiration levels), the sum of aspiration levels is optimal, that is $D=\nu(N)$. We shall now describe how the transitions yielding a reduction in $D$ take place within a (subcomponent. Consider a (sub-)component such that $|\mathcal{F}|>|\mathcal{W}|$ and all firms have positive aspiration levels (the analysis is analogous for the case where there are more workers than firms). By a straightforward argument one sees that there exists at least one firm $i$ which constitutes a leaf of the tree (i.e., only has one edge, say towards worker $j$ ). Suppose we are in a phase of worker-favoring market sentiment. Then, in expectation, after $N$ steps worker $j$ is activated and gets matched with firm $i$ and receives an additional unit. (Note that $j$ may be matched with any firm such that he receives an additional unit; we would just follow a different path.) Recall that in the meantime, given that workers are currently price sticky (market sentiment is worker-favoring) no worker has reduced his aspiration level, hence it still holds that no firm $i^{\prime} \neq i$ has new tight edges. Players who are not at a leaf in the component have two tight edges and hence no renegotiations can take place. In a next step there is a new firm which 
has only one edge (new leaf of a tree) which can meet its corresponding worker and give up $\delta$. (Since it is a period of worker-favoring market sentiment and thus workers are currently price sticky such transitions cannot be reversed.) In total this can happen at most $N$ times. Hence after $O\left(N^{2}\right)$ iterations the sum of aspiration levels is reduced by $\delta$ when the component has more firms than workers. However, it may be that the only (sub-) component without a perfect matching has more workers than firms. In this case we may have to wait $O\left(N^{2+c}\right)$ steps until market sentiment changes and above transitions can take place. It follows that the total time until $D$ is reduced is $O\left(N^{2+c}\right)$.

Lemma 6. Given a state as in Lemma 3, the expected waiting time until $D^{t}=\nu(V)$ is $O\left(N^{2+c}\right.$. $\left.\frac{1}{\delta} \cdot\left(N \cdot w^{*}-\nu(V)\right)\right)$.

Proof A crude bound for the maximal aspiration level of players is given by $w^{*}$ and thus $2 N$. $w^{*}$ for the sum of aspiration levels. By Lemma 3 it follows that $\sum_{i \in V} d_{i} \geq \nu(V)$. Thus the maximal reduction is $O\left(\frac{1}{\delta}\left(N \cdot w^{*}-\nu(V)\right)\right)$. The result now follows from Lemma 5 .

Next, we show by contradiction that from now on for all optimal pairs we have that the edge between them is tight (Lemma 7). It follows that the current aspiration levels are already corresponding to a core allocation. Thus only matchings, break-ups and rematchings are necessary in order to reach the core.

Lemma 7. Given a state $\left[M^{T}, \mathbf{d}^{T}, \Psi^{T}\right]$ as in Lemma 6 let $M$ be the optimal matching (which is unique by tie-freeness). Then for all $(i, j) \in M$ and for all $t \geq T, d_{i}^{t}+d_{j}^{t}=w_{i j}$.

Proof Let $M$ be the optimal matching. Suppose there exists $(i, j) \in M$, such that $d_{i}^{t}+d_{j}^{t}>w_{i j}$. By Lemma 3 we know that (in particular) for all $\left(i^{\prime}, j^{\prime}\right) \in M, d_{i^{\prime}}^{t}+d_{j^{\prime}}^{t} \geq w_{i^{\prime} j^{\prime}}$. Hence

$$
\begin{aligned}
D=\sum_{\left(i^{\prime}, j^{\prime}\right) \in M} d_{i^{\prime}}^{t}+d_{j^{\prime}}^{t} & =d_{i}^{t}+d_{j}^{t}+\sum_{\left(i^{\prime}, j^{\prime}\right) \neq(i, j),\left(i^{\prime}, j^{\prime}\right) \in M} d_{i^{\prime}}^{t}+d_{j^{\prime}}^{t} \\
& \geq d_{i}^{t}+d_{j}^{t}+\sum_{\left(i^{\prime}, j^{\prime}\right) \neq(i, j),\left(i^{\prime}, j^{\prime}\right) \in M} w_{i^{\prime} j^{\prime}} \\
& >w_{i j}+\sum_{\left(i^{\prime}, j^{\prime}\right) \neq(i, j),\left(i^{\prime}, j^{\prime}\right) \in M} w_{i^{\prime} j^{\prime}} \\
& =\sum_{\left(i^{\prime}, j^{\prime}\right) \in M} w_{i^{\prime} j^{\prime}} \\
& =\nu(V)
\end{aligned}
$$

This constitutes a contradiction to the assumption that $D=\nu(V)$ and the result follows.

In order to prove Lemma 8 we first provide a series of supporting lemmas concerning the expected meeting time of two random walks on two, possibly different, sub-trees of a given tree. Our proof technique mirrors Tetali and Winkler (1991) who give a bound for the meeting time of two random walks on (the same) general graph. They show that the expected meeting time on a general graph on $N$ vertices is at most $\frac{8}{27} N^{3}$. 
Support Lemma B. Let $T$ be an undirected tree on $N$ vertices. Let two tokens $x, y$ be placed on two vertices at time $s=0$. Suppose that at each time step $s=1,2,3, \ldots$ one of the two tokens is activated (which token is chosen may be governed by any probability distribution). The token performs a simple random walk to a neighboring vertex on a truncated tree $T_{x}^{s} \subset T$ (or $T_{y}^{s} \subset T$ ). Then, regardless of the rule of activation, if in every time step the (unique) path between $x$ and $y$ is in $T_{x}^{s}$ (respectively $T_{y}^{s}$ ) the expected time $M_{\text {truncated }}(x, y)$ until the tokens meet is $O\left(N^{2}\right)$.

In order to prove the lemma we shall need the following two lemmas from Tetali and Winkler (1991) which can be proved by a straightforward calculation due to the reversibility of the Markov chain for random walks on undirected graphs. Given a connected graph $T$, let $H_{T}(x, y)$ be the hitting time from $x$ to $y$ on $T$, that is the expected time until $y$ is first reached when starting a simple random walk at $x$ on the graph $T$.

Support Lemma C. (Tetali and Winkler, 1991, Lemma 2) Let $x, y$ and $z$ be vertices of a connected undirected graph $T$. Then

$$
H_{T}(x, y)+H_{T}(y, z)+H_{T}(z, x)=H_{T}(x, z)+H_{T}(z, y)+H_{T}(y, x)
$$

Support Lemma D. (Tetali and Winkler, 1991, Lemma 3) On any graph $T$ the vertex relation given by

$$
x \leq y \Longleftrightarrow H_{T}(x, y) \leq H_{T}(y, x)
$$

is transitive.

It immediately follows that, in a finite graph, there exists a vertex $v$ which is minimal in this order. Call such a vertex remote and note that we have $H_{T}(x, v) \geq H_{T}(v, x)$ for every vertex $x \in T$.

Proof (Proof of Support Lemma B) The proof mirrors Tetali and Winkler (1991). We shall show that $\forall x, y \in T$

$$
M_{\text {truncated }}(x, y) \leq H_{T}(x, y)+H_{T}(y, v)-H_{T}(v, y) .
$$

where $v$ is a remote vertex. Define a potential function

$$
\begin{aligned}
P(x, y) \quad & H_{T}(x, y)+\overbrace{H_{T}(y, v)-H_{T}(v, y)}^{\geq 0 \text { since } v \text { is remote }} \\
\text { Support Lemma C } & \begin{array}{c}
= \\
H_{T}(y, x)+H_{T}(x, v)-H_{T}(v, x) .
\end{array}
\end{aligned}
$$

Suppose that the token on $x$ is moved in the next period. Then, in expectation

$$
P(\bar{x}, y)=H_{T}(\bar{x}, y)+H_{T}(y, v)-H_{T}(v, y)
$$

where $P(\bar{x}, y):=\frac{1}{\operatorname{deg}(x)} \sum_{z \in \text { neigh }(x)} P(z, y)$ and $P(x, \bar{y})$ is defined analogously. ${ }^{5}$

Since one move has passed we have

$$
P(x, y)=1+P(\bar{x}, y)
$$

By a symmetrical argument we also have

$$
P(x, y)=1+P(x, \bar{y})
$$

\footnotetext{
${ }^{5}$ We define $\operatorname{deg}(x)$ to be the degree of node $x$ in $T$ and neigh $(x)$ to be the set of neighbors of node $x$ in $T$.
} 
Hence, independent of which token is moved we have that the expected value after the move will decline by one

$$
P(x, y)=1+P(\bar{x}, y)=1+P(x, \bar{y}) .
$$

Define

$$
\beta(x, y)=M_{\text {truncated }}(x, y)-P(x, y) .
$$

Among all pairs maximizing $\beta(\cdot, \cdot)$ choose $x^{*}, y^{*}$ at minimal distance from each other. Now suppose inequality (20) does not hold for all $x, y \in T$, that is $\beta\left(x^{*}, y^{*}\right)>0$ (note that $x^{*} \neq y^{*}$ follows, for otherwise $\left.M_{\text {truncated }}\left(x^{*}, y^{*}\right)=0 \leq P\left(x^{*}, y^{*}\right)\right)$. W.l.o.g. assume that the token moved next is on $x^{*}$. Then

$$
\begin{aligned}
M_{\text {truncated }}\left(x^{*}, y^{*}\right) & =P\left(x^{*}, y^{*}\right)+\beta\left(x^{*}, y^{*}\right) \\
& =1+P\left(\overline{x^{*}}, y^{*}\right)+\beta\left(x^{*}, y^{*}\right) \\
& =1+\frac{1}{\operatorname{deg}\left(x^{*}\right)} \sum_{x_{i}: x_{i}} P\left(x_{i}, y^{*}\right)+\beta\left(x^{*}, y^{*}\right) \\
& >1+\frac{1}{\operatorname{deg}\left(x^{*}\right)} \sum_{x_{i}: x_{i}} P\left(x_{i}, y^{*}\right)+\beta\left(x_{i}, y^{*}\right) \\
& =1+\frac{1}{\operatorname{deg}\left(x^{*}\right)} \sum_{x_{i}: x_{i} \text { neighbor of } x^{*}} M_{\text {truncated }}\left(x_{i}, y^{*}\right) \\
& =M_{\text {truncated }}\left(x^{*}, y^{*}\right)
\end{aligned}
$$

Inequality (31) holds since, first, by the choice of $x^{*}, y^{*}$ for all $i, \beta\left(x_{i}, y^{*}\right) \leq \beta\left(x^{*}, y^{*}\right)$ and, second, there exists at least one $j$ such that $x_{j}$ is closer to $y^{*}$ than $x^{*}$ in the tree. For the latter $x_{j}$ we know that $\beta\left(x_{j}, y^{*}\right)<\beta\left(x^{*}, y^{*}\right.$ ) (since we assumed $x^{*}, y^{*}$ to be at minimal distance among all pairs maximizing $\beta(\cdot, \cdot))$.

But $M_{\text {truncated }}\left(x^{*}, y^{*}\right)>M_{\text {truncated }}\left(x^{*}, y^{*}\right)$ constitutes a contradiction and thus proves inequality (20). By Georgakopoulos and Wagner (2017, Corollary 8) the hitting time on a tree is bounded above by the square of the number of edges $(N-1)$. Thus we have

$$
M_{\text {truncated }}(x, y) \leq H_{T}(x, y)+H_{T}(y, v)=O\left(N^{2}\right) .
$$

Support Lemma E. Let $T$ be a tight component (a tree) for which a perfect matching exists. Suppose the dynamic is in a state such that there are exactly two remaining singles $i$ and $j$. Then after a finite number of transitions where $i$ remained single throughout $i$ matches with some $j^{\prime}$.

Proof We shall show that the path in the component between $i$ and $j$ must be alternating between unmatched and matched edges. Suppose, by contradiction, that the path between $i$ and $j$ is not alternating between unmatched and matched edges. If $i$ and $j$ share an edge we are done. Else, let $i^{*}$ be the first firm (other than $i$ ) on the (unique) path from $i$ to $j$ such that $i^{*}$ is not matched to a worker on the path. (Note that $i^{*}$ is matched elsewhere.) Let $j^{*}$ be the worker preceding $i^{*}$ on the path. We shall construct an instance of Support Lemma A. Delete $i^{*}$ from $T$. Then the component including $i$ is such that all matches are within the component (since $i^{*}$ is matched elsewhere).

Since this component does not contain $j$ and contains $i$ and otherwise only contains matched pairs it is of odd size. On the other hand let $j^{* *}$ be matched with $i^{*}$. Now consider the component containing $j^{* *}$ in $T-i^{*}$. Since $j^{* *}$ is matched to $i^{*}$ it also must be odd. This contradicts 
Lemma Support Lemma A and, by contradiction, establishes the claim that there is an alternating path of unmatched and matched edges between $i$ and $j$.

Let the unique path be $i, j_{1}, i_{1}, j_{2}, i_{2}, \ldots, j_{k}, i_{k}, j$. We can now construct a path, proving the lemma. Suppose $i$ is activated and matches with $j_{1}$, leaving $i_{1}$ single. Next $i_{1}$ is activated and matches with $j_{2}$, leaving $i_{2}$ single. Continuing in this manner we see that after $k$ steps $i_{k}$ is activated and matches with $j$.

We are now in a position to proof the following lemma. In order to give a bound on the expected time until matchings occur we create a duality with random walks on trees. We 'give' each single player a single token which is moved among the players as play unfolds. The idea is that the random walk governing the single tokens will behave in the same way as the underlying model. That is if a player has a single token he is indeed single. If a single player $i$ matches a player from the other side of the market $j$, the single token is moved to $j$ 's previous match. With the above we have a bound for the meeting time of two random walks on (different) trees. By carefully coupling the movements in our model with the two random walks we show that the expected time until the core is reached from a state as discussed above is $O\left(N^{4}\right)$.

Lemma 8. Given a state as in Lemma 6, the expected time until the core is reached is $O\left(N^{4}\right)$.

Proof Given $d_{i}^{t}+d_{j}^{t} \geq w_{i j}$ for all $i, j$, rematches only occur when at least one player is single. Thus the number of matches is weakly increasing over time and it suffices to bound the expected time until two singles match. It follows immediately from Lemma 7 that each tight component allows a perfect matching. We shall compute the expected time until the number of singles is zero. We describe the transitions without taking market sentiment into account since it would only accelerate the process.

We shall construct two simple random walks which we then relate to our process. Let $X, Y$ be two simple random walks on truncated subgraphs of $T$ as defined in Support Lemma B and let $s=0,1,2,3, \ldots$ be the time steps at which one of the random walks moves on some truncated tree (such that the path between the two random walks is on the truncated tree). Let the rule of activation for the random walks be such that both random walks always move twice consecutively. That is, for all $s$ odd, if $X$ (respectively $Y$ ) is activated in $s$, then $X$ (respectively $Y$ ) is also activated in $s+1$. Define for $X$ (analogously for $Y$ ) the following time steps. Initialize $T_{0}^{X}=T_{0}^{Y}=0$, then for $i=1,2,3, \ldots$ let

$$
T_{i}^{X}=\inf \left\{s>T_{i-1}: s \text { odd, and } X_{s} \neq X_{s-2}\right\},
$$

and

$$
\widetilde{T}=\inf \left\{s: X_{s}=Y_{s}\right\} .
$$

The sequence of time steps at which the random walk $X$ (or $Y$ ) has moved away from odd $s$ after two time steps or has collided with $Y$ (respectively $X$ ) is given by

$$
\begin{aligned}
& \mathcal{T}^{X}=\left(T_{1}^{X}, T_{2}^{X}, \ldots, T_{I}^{X}, \widetilde{T}\right), \\
& \mathcal{T}^{Y}=\left(T_{1}^{Y}, T_{2}^{Y}, \ldots, T_{J}^{Y}, \widetilde{T}\right),
\end{aligned}
$$

where $I, J$ are two random variables describing the lengths of the sequences. Note that for all time-steps $s \notin \mathcal{T}^{X} \cup \mathcal{T}^{Y}$ the random walk returned to its previous place after two steps. Define $\widetilde{X}$ and $\widetilde{Y}$ to be the (not simple) random walks of step-size two on truncated trees. Until the two underlying random walks $X$ and $Y$ collide, $\widetilde{X}$ and $\widetilde{Y}$ move at times $\mathcal{T}^{X} \cup \mathcal{T}^{Y}$. 
We now relate the two random walks, $\tilde{X}, \tilde{Y}$, to our process. We shall first consider the case where only two singles remain in a given tight component. Suppose $i$ and $j$ are the only two remaining singles. Note that the two singles will always remain in the same tight component. Now consider those time steps where a current single becomes matched and a previously matched player becomes single. (The expected interval between such time steps is upper bounded by $O(N)$.)

Suppose we start the random walk $\widetilde{X}$ on the single firm $i$ and the random walk $\widetilde{Y}$ on the single worker $j$. Suppose $i$ (or $j$ ) gets activated in a given time step. Then $\widetilde{X}$ (or $\widetilde{Y}$ ) is also activated and moves to a node in the tree at distance two from $i$, say $i^{\prime}$ (via $\left.j^{\prime}\right)$ such that $\left(i^{\prime}, j^{\prime}\right.$ ) were matched in the previous period. This is the condition for the truncated tree on which the random walk $\widetilde{X}$ (or $\widetilde{Y}$ ) moves, namely the truncated tree starting at $i$ only has branches alternating between tight unmatched and matched edges. Any such node $i^{\prime}$ is picked uniformly at random amongst the matches of current neighbors. Thus $\widetilde{X}$ (respectively $\widetilde{Y}$ ) perform the previously defined two-step random walk. The time steps at which this happens are precisely given by $\mathcal{T}^{X}$ (respectively $\mathcal{T}^{Y}$ ). This indicates that player $i$ is matching with player $j^{\prime}$. Now if $j^{\prime}=j$ the two last remaining singles match and the simple random walks $X$ and $Y$ collide. Else $i^{\prime}$ is now occupied by $\widetilde{X}$.

We are left to give an upper bound for $\mathbb{E}[I+J+1]$. First note that we have $\mathbb{E}[I+J+1]<$ $M_{\text {truncated }}(X, Y)$. We shall now argue that

$$
\mathbb{E}[I+J+1]=\frac{1}{4} M_{\text {truncated }}(X, Y)
$$

and thus the bound we shall give is sharp. If $X$ (or $Y$ ) is moved in periods $s+1$ (odd) and $s+2$ we have $\mathbb{P}\left(X_{s+2}=X_{s}\right)=1 / 2$. This is the case since on the truncated tree the random walk first moves along a tight edge and then necessarily moves back or along a matched edge. Thus $\mathbb{E}\left[T_{i+1}^{X}-T_{i}^{X}\right]=4$ given the expectation of the geometric distribution with parameter $1 / 2$. Now the result follows.

Overall, given the singles need to be activated in order for the random walks to be moved, the meeting of the two single tokens takes $O\left(N^{3}\right)$ iterations.

Now suppose there are $2 k(k \in\{1, \ldots, N\})$ remaining singles. The expected time until there are only $2(k-1)$ singles is bounded by $O\left(N^{3}\right)$. Then for $k=O(N)$ we have a total expected time until the core is reached of $O\left(N^{4}\right){ }^{6}$

Finally, it is easy to see that a single iteration has computational complexity $O\left(N^{2}\right)$ (Lemma $9)$.

Lemma 9. One iteration of the dynamic takes $O\left(N^{2}\right)$ operations.

Proof Given that $i$ is activated, suppose there exists a feasible match. The calculation whether a player has no feasible alternative requires $O(N)$ for each $j^{\prime}=1, \ldots, N$, and thus $O\left(N^{2}\right)$ in total. The case where no feasible match exists for $i$ requires at most $O(N)$ operations. The result now follows.

Putting together the different bounds, we can finally proof the proposition:

\footnotetext{
${ }^{6}$ In line with Tetali and Winkler (1991, Conjecture 2) we conjecture that this bound is not optimal and the total meeting time of $k$ random walks on truncated trees is of the same order as the two-token meeting time. Together with the necessary activation of single tokens this would yield the bound $O\left(N^{3}\right)$.
} 
Proof (Proof of Proposition 2) Finally considering the bounds found in Lemmas 3, 6, and 8:

$$
\begin{aligned}
& \text { Lemma } 3: O\left(N^{2} \log N\right) \\
& \text { Lemma } 6: O\left(N^{2+c} \cdot \frac{1}{\delta} \cdot\left(N \cdot w^{*}-\nu(V)\right)\right. \\
& \text { Lemma } 8: O\left(N^{4}\right)
\end{aligned}
$$

we have that the bound in Lemma 8 strictly dominates the bound in Lemma 3 . We can thus conclude that the expected maximum number of steps to absorption is

$$
O\left(N^{2+c} \cdot \max \left\{N^{2}, \frac{1}{\delta}\left(N \cdot w^{*}-\nu(V)\right)\right\}\right)
$$

proving the first part of the theorem. By Lemma 9 we know the complexity of each iteration, $O\left(N^{2}\right)$, and the second part of the theorem follows.

\section{Results without market sentiment}

Next, we investigate the case when there is no alternating market sentiment. We shall find that convergence per se is still guaranteed without market sentiments (Proposition 10), but convergence can be exponentially slow (Proposition 11).

Proposition 10. For any assignment game, decentralized dynamics based on aspiration adjustment converge to the $\varepsilon$-core.

Proof The proof of convergence to the approximate core in the absence of the tie-free condition and the market sentiment follows from the proof of Proposition 2 by the following observations. In particular, note that the proof of Lemma 5 only uses tie-freeness and the market sentiment to establish polynomial bounds; for convergence those properties are not needed. The other supporting lemmas do not use the conditions at all. Note that Proposition 2 shows convergence to the core of the discretized game which is the $\varepsilon$-core for appropriately chosen $\varepsilon$ of the original game.

Proposition 11. Convergence to the $\varepsilon$-core may take in the order of $e^{\Theta(N)}$.

Proof We shall prove this by constructing an example. Suppose we have an assignment game as shown in Figure 1. The unique optimal matching is given by the vertical lines. As pointed out after Definition 1 this game constitutes a tie-free assignment game with since the set of positive-weight edges constitutes a tree and thus Equation 1 never holds.

Suppose we are in the starting state displayed in Figure 2, where the aspiration levels are shown next to each agent. Note that the only edge such that the sum of the aspiration levels exceeds the match value is $\left(i_{0}, j_{0}\right)$.

In order for $i_{0}$ and $j_{0}$ to match at least one of them has to reduce his aspiration level. Recall that a player only reduces his aspiration level if he has no feasible alternative. Thus, for $i_{0}$ or $j_{0}$ to reduce their aspiration level, either all of $i_{r_{1}}, \ldots, i_{r_{(N-1) / 2}}$ or all of $j_{l_{1}}, \ldots, j_{l_{(N-1) / 2}}$ have to increase their aspiration levels simultaneously. If the agents on both sides of the market are not price sticky the sharing between any two (currently matched) agents switches back and forth between sharing equally $(1-1)$ and sharing $0-2$ with the player previously having a 


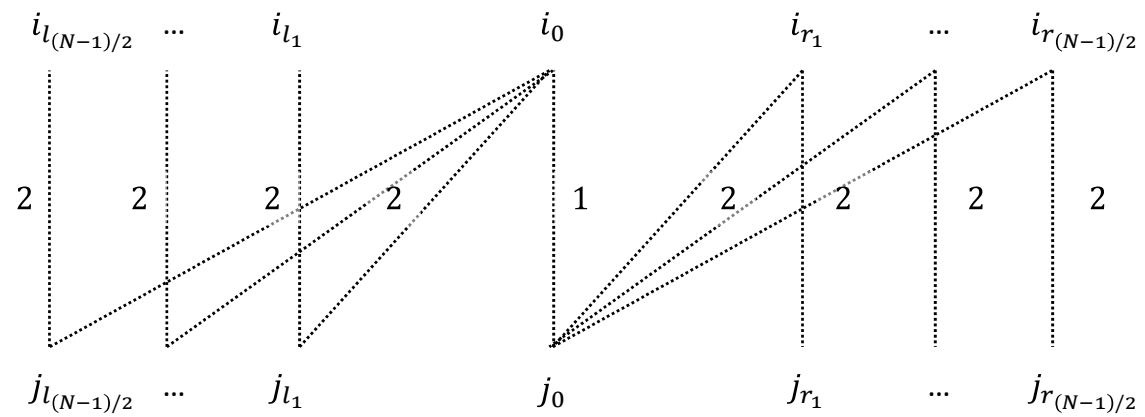

Fig. 1: Counterexample - necessity of alternating market sentiment (1).

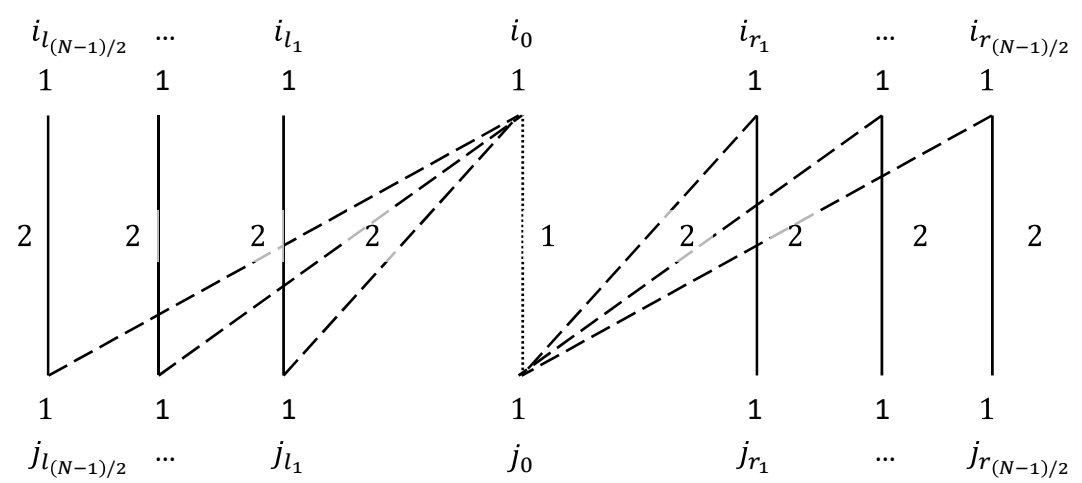

Fig. 2: Counterexample - necessity of alternating market sentiment (2).

tight edge with $i_{0}$ or $j_{0}$ receiving 2 . To see this consider, for example, $i_{l_{1}}$ and $j_{l_{1}}$ with current sharing of $1-1$. In a first step $j_{l_{1}}$ can match with $i_{0}$ at the same price. In the next step, $i_{l_{1}}$ is single and can be forced to give up 1 by $j_{l_{1}}$ since he has no feasible alternative. On the other hand, if the sharing is currently $0-2$ then $j_{l_{1}}$ can be forced to give up 1 by $i_{l_{1}}$ since he has no feasible alternative. The probability that all $i_{r_{1}}, \ldots, i_{r_{(N-1) / 2}}\left(\right.$ or all $j_{l_{1}}, \ldots, j_{l_{(N-1) / 2}}$ ) hold aspiration level 2 at the same time is of order $\frac{1}{2^{N}}$ and therefore the waiting time until either $i_{0}$ or $j_{0}$ reduce their aspiration level is exponential and is $e^{\Theta(N)}$.

\section{Conclusion}

We have shown that decentralized market dynamics generally converge to the approximate core, and we have obtained first results for the rates of convergence that may be obtained. We have shown that, even if markets are large, convergence may be sped up for tie-free games when dynamics are governed by what we called 'market sentiments' that make one or the other of the two market sides amenable to price reductions, and that this sentiment changes sides at a certain (not too fast and not too slow) rate. Both the presence of market sentiments and tie-freeness are crucial for our results. Without market sentiment, our dynamic may take ex- 
ponentially long to converge. ${ }^{7}$ Our analysis indicates that many realistic market (i.e. ones that are finite, tie-free, and characterized by market sentiments with strongly heterogeneous preferences) may converge to competitive equilibrium fast. Indeed, we view our results as a first step in the direction of confirming - by use of convergence rate arguments - that the core is predictively relevant for decentralized markets as existent in the real-world as well. It is an avenue for future research to establish whether better conditions and bounds for convergence can be established without market sentiment, independently of the discretization, and for more general classes of games.

Acknowledgements Pradelski thanks Pierre Tarrès and Peyton Young for their guidance and support throughout and beyond his PhD which shaped this research. Further, this paper has benefitted from generous comments and criticism by Yakov Babichenko, Doyne Farmer, Satoru Fujishige, Olivier Gossner, Sergiu Hart, Michael Krivelevich, Jacob Leshno, Noam Nisan, Thomas Norman, Bill Sandholm, Jay Sethuraman, Eran Shmaya, Gregory Sorkin and Bernhard von Stengel, as well as from anonymous referees. All of their comments are gratefully acknowledged. Comments by participants at seminars at Hebrew University (Israel), Technion (Israel), University of Oxford (UK), MEDS, Northwestern (Evanston), DRO, Columbia Business School, Microsoft Research, New England, EC' 15, Portland, GAMES 2016, Maastricht, IMA, OR Society Conference on Mathematics of Operational Research 2017, Paris Game Theory Seminar, and Ecole Polytechnique were also important, and we thank all of them.

\section{References}

Ackermann H, Goldberg P, Mirrokni V, Roeglin H, Voecking B (2011) Uncoordinated twosided matching markets. SIAM Journal on Computing 40(1):92-106

Arieli I, Young HP (2016) Stochastic learning dynamics and speed of convergence in population games. Econometrica 84:627-676

Bayati M, Borgs C, Chayes J, Kanoria Y, Montanari A (2015) Bargaining dynamics in exchange networks. Journal of Economic Theory 156:417-454

Biró P, Bomhoff M, Golovach PA, Kern W, Paulusma D (2012) Solutions for the stable roommates problem with payments. Lecture Notes in Computer Science 7551:69-80

von Böhm-Bawerk E (1891) The positive theory of capital. London; New York: Macmillan and Company

Chen B, Fujishige S, Yang Z (2016) Random decentralized market processes for stable job matchings with competitive salaries. Journal of Economic Theory 165:25 - 36

Edmonds J (1965) Paths, trees, and flowers. Canadian Journal of Mathematics 17:49-467

Feller W (1950) An introduction to probability theory and ist applications. John Wiley

Gale D, Shapley LS (1962) College admissions and stability of marriage. American Mathematical Monthly 69:9-15

Georgakopoulos A, Wagner S (2017) Hitting times, cover cost, and the Wiener index of a tree. Journal of Graph Theory 84(3):311-326

Klaus B, Payot F (2015) Paths to stability in the assignment problem. Journal of Dynamics and Games 2:257-287

Leshno JD, Pradelski BSR (2018) Efficient price discovery and information in the decentralized assignment game. Working paper

Lewis D (1969) Convention: A philosophical study. Harvard University Press

Nax HH, Pradelski BSR (2015) Evolutionary dynamics and equitable core selection in assignment games. International Journal of Game Theory 44(4):903-932

\footnotetext{
${ }^{7}$ See also Leshno and Pradelski (2018) who show that there exist assignment games for which any decentralized dynamic with no additional memory or information (such as through market sentiment as is proposed here) takes exponential time to converge.
} 
Newton J, Angus SD (2015) Coalitions, tipping points and the speed of evolution. Journal of Economic Theory 157:172-187

Núñez MNO, Rafels C (2015) A survey on assignment markets. Journal of Dynamics and Games, 2015, Vol 2 (3-4) pp 227-256

Roth AE, Vande Vate H (1990) Random paths to stability in two-sided matching. Econometrica 58:1475-1480

Shapley LS, Shubik M (1972) The assignment game 1: The core. International Journal of Game Theory 1(1):111-130

Tetali P, Winkler P (1991) On a random walk problem arising in self-stabilizing token management. In: Proceedings of the tenth annual ACM symposium on Principles of distributed computing, ACM, New York, NY, USA, PODC '91, pp 273-280 